\title{
STABILITY ANALYSIS UTILIZING AMMI MODEL AND REGRESSION ANALYSIS FOR GRAIN YIELD OF BASMATI RICE (Oryza sativa L.) GENOTYPES
}

\author{
Bharat Taindu Jain $^{1 *}$, Ashok Kumar Sarial ${ }^{2}$, Prashant Kaushik ${ }^{3 *}$ \\ ${ }^{1 *}$ Department of Genetics \& Plant Breeding, CCSHAU, Hisar, Haryana 125001, India \\ ${ }^{2}$ Vice-Chancellor, CSKHPKV, Palampur, Himachal Pradesh 176062, India \\ ${ }^{3 *}$ Instituto de Conservación y Mejora de la AgrodiversidadValenciana, UniversitatPolitècnica de València, Camino de Vera 14, 46022, Valencia, Spain
}

Received - February 12, 2018; Revision - April 28, 2018; Accepted - June 02, 2018

Available Online - June 20, 2018

DOI: http://dx.doi.org/10.18006/2018.6(3).522.530522

KEYWORDS
Rice
Genotypes
G $\times$ E
TPR
SRI
DSR
AMMI biplot analysis

\begin{abstract}
A field experiment comprising of released varieties, elite lines and hybrid under four production systems viz. Transplanted rice (TPR), Dry direct seeded rice (Dry DSR), Wet direct seeded rice (Wet DSR) and System of rice intensification (SRI) was conducted at Research farm of RRS, Kaul, CCS HAU, Hisar, Haryana during Kharif of 2014-15 and 2015-16. Present study tested and identified the basmati rice genotypes for yield and its components under System of rice intensification, Direct seeded rice (both Dry and Wet) and regular Transplanted rice system. Four characters namely, grain yield, plant height, tiller/ plant panicle length and panicle weight were recorded.

Estimates of genotype $\mathrm{x}$ environment interaction and additive main effect were significant for all the mentioned traits. The traits viz. tiller/ plant and plant height were found proportional to grain yield. Based on AMMI biplot analysis genotypes Pusa RH 10 has been identified for SRI, while genotypes i.e. Imp Pusa Basmati-1, CSR-30, Haryana Basmati-1 and Haryana Mehak-1 were identified for Wet DSR and two genotypes Super Basmati and HKR 06-487 for Dry DSR conditions.
\end{abstract}

* Corresponding author

E-mail: jainbharat91@gmail.com (Bharat Taindu Jain); prashantumri@gmail.com (Prashant Kaushik)

Peer review under responsibility of Journal of Experimental Biology and Agricultural Sciences.

Production and Hosting by Horizon Publisher India [HPI] (http://www.horizonpublisherindia.in/).

All rights reserved.
All the article published by Journal of Experimental Biology and Agricultural Sciences is licensed under a Creative Commons Attribution-NonCommercial 4.0 International License Based on a work at www.jebas.org.

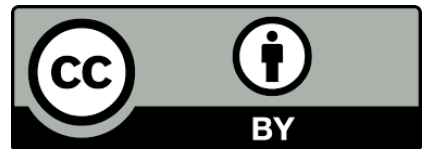




\section{Introduction}

Rice (Oryza sativa L.) is such a wonder crop as it can be grown under diversified edaphic conditions like aerobic and anaerobic (Submerged Conditions)(Miro \& Ismail, 2013). In the present era of energy crisis and the inadequacy of water, it is not possible to proceed for transplanted rice (Nguyen \& Ferrero, 2006). Due to pressure exerted by input crisis, rice production systems are experiencing great changes like wet direct seeded rice, dry direct seeded rice, drum seeding and system of rice intensification (Dawe, 2010; Prasetyo \& Anindita, 2016). For the success of alternate non-conventional resources, the saving techniques and optimization of input requirement are mandatory (Rao et al., 2017). To date, the researchers have been focused on optimum input requirement for transplanted rice, however, the new production systems are governed by the inputs fixed for transplanted rice or a slight change in a previous recommendation. Thus, it is not possible to exploit the complete potential of genotypes under non-conventional methods, which is one of the major hurdles for low adoption rate of these methods among rice growers. Evaluation of different genotypes performance under different production systems is required for the optimization of the genetic potential of different genotypes under diverse edaphic conditions. Since conventional transplanting system during puddling creates a hard pan below the plough-zone, reduces soil permeability and increases water loss through surface evaporation and percolation. Because of high water requirement and labor cost profits are reduced (Surendra, 2010). Therefore, in recent years, the farmers have shifted from a conventional to a nonconventional method of paddy cultivation, namely the system of rice intensification and direct seeded rice in several countries of Southeast Asia (Takashima et al., 2013). Hence, to promote diversification and enable farmers to choose a crop alternative for increased productivity and income, the technology innovation involving non-conventional production methods such as the system of rice intensification and direct seeded rice in basmati rice cultivation could be explored to enhance the productivity and sustainability in agriculture (Vaughan et al., 2008; Fuller et al., 2016). Uphoff (2003), Satyanarayana et al. (2007) and Grihtlahre et al. (2012) have advocated the adaptation of system of rice intensification and obtained on an average $12-24 \%$ higher grain yield by a system of rice intensification over the normal transplanted system. Therefore, the present experiment was performed to understand the genetics of interaction of rice genotypes with the environment and to identify the stable genotype to sustain new production systems applied to rice these days to conserve resources.

\section{Materials and Methods}

\subsection{Experimental site and design}

A field experiment was laid out during the Kharif season of 20142015 and 2015-2016 at the Regional Research Station Kaul, CCSHAU, Haryana, Hisar India. Geographically it is located at $29.8498^{\circ}$ and $76.6615^{\circ} \mathrm{E}$ longitudes which fall under the subtropical region. The experiments were carried out in a Randomized Complete Block Design (RCBD) with three replications in total seven environments (four production systems in 2014-15 and three production systems in 2015-2016) created agronomically termed production systems of rice as shown in Table 1. Plot size consisted of 5 rows of $2 \mathrm{mtr}$ long and $0.20 \mathrm{mtr}$ row spacing each. The accessions used in the present study were consisted of twenty two popular genotypes grown in the state Haryana (Table 2).

\subsection{Statistical Analysis}

The analysis of variance of the present investigation was carried out as per the standard procedures of Panse \& Sukhatme (1985) for Grain Yield per plant, Plant height, Panicle Length, Panicle Weight, Number of Tillers per Plant in all the seven environments (E1-E7) (Table 1). Standard package of practices was followed for the rice production. Border rows were not included in the data to avoid the border effect. The data for grain yield were taken at the $14 \%$ moisture while all the other remaining characters were measured as demarcated in the manual (Yoshida et al., 1976). Analysis of variance was computed for the individual environment. The significance of all effects was tested against the mean square of error. Data were subjected to statistical analysis using Windostat Version 9.2 (Indostat services, Hyderabad,

Table 1 Environmental conditions used during study

\begin{tabular}{|c|c|c|c|c|c|c|c|}
\hline \multirow[t]{2}{*}{ Environment } & \multicolumn{4}{|c|}{ 2014-2015 } & \multicolumn{3}{|c|}{$2015-2016$} \\
\hline & E1 & E2 & E3 & E4 & E5 & E6 & E7 \\
\hline Description & $\begin{array}{c}\text { Transplanted } \\
\text { Rice (TPR) }\end{array}$ & $\begin{array}{c}\text { System of } \\
\text { Rice } \\
\text { Intensification } \\
\text { (SRI) }\end{array}$ & $\begin{array}{l}\text { Direct Seeded } \\
\text { Rice (DSR) } \\
\text { (Wet) }\end{array}$ & $\begin{array}{l}\text { Direct Seeded } \\
\text { Rice (DSR) } \\
\text { (Dry) }\end{array}$ & $\begin{array}{l}\text { Transplanted } \\
\text { Rice (TPR) }\end{array}$ & $\begin{array}{l}\text { Direct Seeded } \\
\text { Rice (DSR) } \\
\text { (Wet) }\end{array}$ & $\begin{array}{l}\text { Direct Seeded } \\
\text { Rice (DSR) } \\
\text { (Dry) }\end{array}$ \\
\hline
\end{tabular}

Journal of Experimental Biology and Agricultural Sciences http://www.jebas.org 
Table 2 List of Genotypes Used in Study Table is Adapted from Jain et al. (2018).

\begin{tabular}{|c|c|c|}
\hline Code & Genotypes & Pedigree \\
\hline 1 & Pusa Basmati 1121 & P614-1-2/P614 2-4-3 \\
\hline 2 & Pusa Basmati 1509 & PUSA 1301/PB1121 \\
\hline 3 & Pusa Sugandh 2 & PUSA 1238-1/PUSA 1238-81-6 \\
\hline 4 & Pusa Sugandh 3 & PUSA 1238-1/PUSA 1238-81-6 \\
\hline 5 & Pusa Sugandh 5 & PUSA 3A/ HARAYANA BASMATI \\
\hline 6 & Pusa Basmati 6 & PUSA BASMATI-1/PUSA 1121-92-8-2-7-1 \\
\hline 7 & Pusa Basmati 1 & PUSA 150/ KARNAL LOCAL \\
\hline 8 & Improved Pusa Basmati 1 & PB 1//PB 1/ IRBB55 \\
\hline 9 & HKR 98-476 & Collected from RRS, Kaul, Haryana \\
\hline 10 & HKR 03-408 & Collected from RRS, Kaul, Haryana \\
\hline 11 & HKR 06-434 & Collected from RRS, Kaul, Haryana \\
\hline 12 & HKR 06-443 & Collected from RRS, Kaul, Haryana \\
\hline 13 & HKR 06-487 & Collected from RRS, Kaul, Haryana \\
\hline 14 & HKR 08-417 & Collected from RRS, Kaul, Haryana \\
\hline 15 & HKR 08-425 & Collected from RRS, Kaul, Haryana \\
\hline 16 & Haryana Mahek-1 & Collected from RRS, Kaul, Haryana \\
\hline 17 & Haryana Basmati-1 & SONA/BASMATI 370 \\
\hline 18 & Traori Basmati & SELECTION BASMATI 370 \\
\hline 19 & Super Basamati & BASMATI 320/IR661 \\
\hline 20 & CSR-30 & BR4-10/BASMATI-370 \\
\hline 21 & Basmati-370 & OLD SELECTION \\
\hline 22 & Pusa RH-10 & PUSA 6A/PRR 78 \\
\hline
\end{tabular}

India). The performance of twenty-two genotypes was tested over four production system for two year and analyses using stability models viz, (1) Eberhart \& Russel (1966), (2) Additive Main effects and Multiplicative Interaction (AMMI) (Gauch \& Zobel, 1989).

\section{Results and Discussion}

\subsection{Genotypic performance of Yield and Yield Related Traits}

In four different production systems-wide genotypic variation was observed and average pooled data ranged for Plant Height (75.00-116.00); Tiller per Plant (10.21-14.51); Panicle Length (22.66-25.33); Panicle Weight (1.08-2.45); Grain Yield Per Plant (8.74-15.25) among the twenty-two genotypes under study as shown in Table 5. Considering the four-different production system among twenty-two genotypes, Pusa RH 10 scored high Grain yield per plant $(15.25 \mathrm{~g})$, Panicle Length $(24.93 \mathrm{~cm})$ and Panicle Weight (22.30 g). CSR-30 recorded the minimum Grain Yield per plant in all production systems except System of Rice Intensification in which it performed better while HKR 06-434 had the maximum (17.11 g) Grain yield per plant in Transplanted Rice, Pusa Basmati 6 (28.41 g) followed by Pusa Basmati 1121 $(27.16 \mathrm{~g})$ in System of Rice Intensification, Pusa RH 10 in Direct Seeded Rice (wet) (18.04 g) and Direct Seeded Rice (dry)(14.22). Pusa RH 10 consistently yielded better in all production systems. In DSR (dry) only three genotypes Pusa Sugandh 5, Traori Basmati and Pusa RH 10 performed better. Genotypic performance of rice in realtion to different environments has been

\section{Journal of Experimental Biology and Agricultural Sciences} http://www.jebas.org 
estimated by worker like Mohammadi et al. (2012), Rakshit et al. (2012) and Amiri et al.(2015) all of them identified verities suitable for a defined production system.

\subsection{Pooled analysis of variance}

The ANOVA showed that mean squares due to genotypes were highly significant for grain yield in all environments. The significance of genotypes mean squares indicated that genotypes differed among themselves and there existed a considerable variability irrespective of the effect of environments on the characters under study. Inconsistent performance of a given cultivar in different environments is termed as $\mathrm{G} \times \mathrm{E}$ interaction. Pooled analysis of variance combined with joint regression analysis proposed by Yates \& Cochran (1938), modified by Finlay \&Wilkinson (1963) and made popular by Eberhart \&Russell (1966) has been and still is a popular technique for studying $\mathrm{G} \times \mathrm{E}$ effects and stability. Additive main effects and multiplicative interaction (AMMI), GGE Biplot analysis is extensively used for Genotype-environment interaction investigation.

The pooled analysis (Table 3 ) was highly significant $(\mathrm{p}<0.01)$ for genotypes and environments for five characters except for Panicle length which it was non-significant for genotype. This revealed that there was considerable variability among genotypes and production systems. The mean squares due to $G \times E$ interaction when tested against pooled error were significant for all the characters viz; Grain yield per plant, Tiller per plant, Panicle different production systems. Sinha \& Biswas (1987) also reported significant $\mathrm{G} \times \mathrm{E}$ interaction for Grain Yield per plant, but not for tiller/plant.

\subsection{Stability Estimates}

Both the parameters i.e. bi and S- 2dimentioned in the model of Eberhart \& Russel (1966) were used to define stability of our genotypes. The stability analysis revealed one genotype in case of Plant height, Panicle length three genotypes in Panicle weight, two genotypes in Grain yield per plant and Tiller per plant had significant regression coefficient (bi) and non-significant deviation from the regression $\left(\mathrm{S}^{-2} \mathrm{di}\right)$ in Table 5. Genotype Pusa Basmati-6 in Grain yield per plant, Pusa Sugandh 2 in Panicle Weight, Haryana Basmati-1 in Panicle Length, Pusa Basmati 1121 in Tiller Per Plant, HKR 98-476 in Plant Height exhibited nonsignificant $\mathrm{S}^{-2} \mathrm{di}$, regression coefficient significantly greater than one, and mean greater than the population mean was found suitable for a better environment. The better environment here refers to Transplanted Rice and System of Rice Intensification. Genotype Pusa RH 10 in Panicle Weight with regression coefficient significantly less than one and non-significant deviation from regression and mean greater than the population mean was identified suitable for unfavorable environments Direct Seeded rice (dry \& wet). None of the genotypes was found stable. Moreover in the previous studies it is well demonstrated that the yield from DSR was low than puddled rice overall stability of DSR was not as much of other systems like SRI when it comes to

Table 3 Pooled Analysis of variance over 7 environments (production systems) for yield related traits in rice (Eberhart \& Russell, 1966 model)

\begin{tabular}{|lcrcccc|}
\hline Source of Variations & DF & Grain Yield & Plant Height & Tiller/Plant & Panicle Length & Panicle weight \\
\hline Rep within Env. & 14 & 0.3 & 7.37 & 0.43 & 0.34 & 0.01 \\
\hline Varieties & 21 & $16.58^{* *}$ & $831.44^{* *}$ & $8.96^{* *}$ & 3.1779 & $0.76^{* *}$ \\
\hline Env.+ (Var.* Env.) & 132 & $23.44^{* *}$ & $170.03^{* *}$ & $10.26^{* *}$ & $3.46^{*}$ & $0.23^{* *}$ \\
\hline Environments & 6 & $326.74^{* *}$ & $2779.87^{* *}$ & $139.28^{* *}$ & $27.65^{* *}$ & $3.29^{* *}$ \\
\hline Var.* Env. & 126 & $9.005^{* *}$ & $45.75^{* *}$ & $4.12^{* *}$ & 2.3149 & $0.087^{*}$ \\
\hline Environments (Lin.) & 1 & $1960.49^{* *}$ & $16679.26^{* *}$ & $835.68^{* *}$ & $165.93^{* *}$ & $19.78^{* *}$ \\
\hline Var.* Env.(Lin.) & 21 & $18.15^{* *}$ & 42.0537 & $6.48^{*}$ & 1.9105 & $0.173^{* *}$ \\
\hline Pooled Deviation & 110 & $6.84^{* *}$ & $44.38^{* *}$ & $3.48^{* *}$ & $2.28^{* *}$ & $0.066^{* *}$ \\
\hline Pooled Error & 294 & 0.7831 & 7.4935 & 0.7892 & 0.4354 & 0.0197 \\
\hline Total & 153 & 22.5065 & 260.814 & 10.0855 & 3.4271 & 0.3064 \\
\hline
\end{tabular}

*, ** Significant at $5 \%$ and $1 \%$ respectively

Weight, Panicle Length, Panicle Height indicating that genotypes interacted with environments and performed inconsistently over 
Indo-Gangetic Plains with alluvial soils compared to other parts of country (Pandey \&Velasco, 2002; Raman et al., 2011).

\subsection{AMMI analysis}

AMMI models are well used to study the stability statistics and helped at several instances to indentify genotypes for a broad environments.

3.4.1 1st year (2014-2015) and 2nd year (2015-2016)

ANOVA based on AMMI as shown in Table 4. This has shown

Table 4 AMMI analysis of Rice genotypes over different production system for 2014-15 and 2015-16 Analyses of Variance for AMMI model

\begin{tabular}{|c|c|c|c|c|c|c|}
\hline \multirow{2}{*}{ Source of Variations } & \multicolumn{3}{|c|}{ 2014-2015 } & \multicolumn{3}{|c|}{$2015-2016$} \\
\hline & D.F. & M.S.S. & Variance $\%$ & D.F. & M.S.S & Variance $\%$ \\
\hline Trials & 87 & $26.306^{*}$ & & 153 & $22.50 * *$ & \\
\hline Genotypes & 21 & $21.158 *$ & & 21 & $16.58 *$ & \\
\hline Environments & 3 & $399.06 *$ & & 6 & $326.76^{* * *}$ & \\
\hline $\mathrm{G}^{*} \mathrm{E}$ Interaction & 63 & $10.27 *$ & & 126 & $9.005^{* *}$ & \\
\hline PCA I & 23 & $17.34 *$ & 61.64 & 26 & $17.83^{* *}$ & $40.87 \%$ \\
\hline PCA II & 21 & $7.1^{*}$ & 23.06 & 24 & $9.85^{* *}$ & $20.85 \%$ \\
\hline PCA III & 19 & $5.2 *$ & 15.28 & 22 & $7.70 * *$ & $14.94 \%$ \\
\hline
\end{tabular}

$*$, ** Significant at $5 \% \& 1 \%$ level of significance, respectively

Table 5 Stability parameters for Yield related trait of rice genotypes tested over 7 environments (production systems) (Eberhart \&Russell, 1966 model)

\begin{tabular}{|c|c|c|c|c|c|c|}
\hline Genotypes & Parameters & Grain Yield & Plant Height & Tiller/ Plant & Panicle Length & Panicle Weight \\
\hline Pusa Basmati 1121 & $\begin{array}{l}\text { Mean } \\
\text { bi } \\
\mathrm{S}^{-2} \mathrm{di}\end{array}$ & $\begin{array}{c}13.552 \\
1.523 \\
6.80^{* *}\end{array}$ & $\begin{array}{c}88.721 \\
0.938 \\
20.82 * *\end{array}$ & $\begin{array}{c}14.514 \\
1.79^{*} \\
0.95\end{array}$ & $\begin{array}{c}24.224 \\
0.497 \\
0.345\end{array}$ & $\begin{array}{c}1.469 \\
0.717 \\
0.01\end{array}$ \\
\hline Pusa Basmati 1509 & $\begin{array}{c}\text { Mean } \\
\text { bi } \\
\mathrm{S}^{-2} \mathrm{di}\end{array}$ & $\begin{array}{c}12.471 \\
0.721 \\
1.71^{* *}\end{array}$ & $\begin{array}{c}84.571 \\
0.959 \\
15.16^{*}\end{array}$ & $\begin{array}{l}12.23 \\
1.334 \\
0.523\end{array}$ & $\begin{array}{c}25.293 \\
1.098 \\
0.277\end{array}$ & $\begin{array}{c}1.833 \\
0.779 \\
0.049 * *\end{array}$ \\
\hline Pusa Sugandh 2 & $\begin{array}{c}\text { Mean } \\
\text { bi } \\
\mathrm{S}^{-2} \mathrm{di}\end{array}$ & $\begin{array}{c}12.827 \\
0.855 \\
4.44 * *\end{array}$ & $\begin{array}{c}87.019 \\
1.019 \\
13.67 *\end{array}$ & $\begin{array}{c}11.14 \\
1.348 \\
3.18^{* *}\end{array}$ & $\begin{array}{c}24.79 \\
1.447 \\
2.02 * *\end{array}$ & $\begin{array}{c}2.457 \\
1.83 \\
0.049^{* *}\end{array}$ \\
\hline Pusa Sugandh 3 & $\begin{array}{c}\text { Mean } \\
\text { bi } \\
\mathrm{S}^{-2} \mathrm{di}\end{array}$ & $\begin{array}{c}12.279 \\
1.037 \\
7.84 * *\end{array}$ & $\begin{array}{c}91.636 \\
0.261 \\
125.77 *\end{array}$ & $\begin{array}{c}10.71 \\
0.889 \\
9.05^{* *}\end{array}$ & $\begin{array}{c}24.078 \\
0.759 \\
0.237\end{array}$ & $\begin{array}{c}1.884 \\
1.269 \\
0.14 * *\end{array}$ \\
\hline Pusa Sugandh 5 & $\begin{array}{c}\text { Mean } \\
\text { bi } \\
\mathrm{S}^{-2} \mathrm{di}\end{array}$ & $\begin{array}{c}12.514 \\
0.648 \\
6.89 * *\end{array}$ & $\begin{array}{c}86.074 \\
0.829 \\
1.829\end{array}$ & $\begin{array}{c}10.4 \\
0.99 \\
1.15^{*}\end{array}$ & $\begin{array}{c}24.247 \\
1.528 \\
0.357\end{array}$ & $\begin{array}{c}2.116 \\
1.319 \\
0.098^{* *}\end{array}$ \\
\hline Pusa Basmati 6 & $\begin{array}{c}\text { Mean } \\
\text { bi } \\
\mathrm{S}^{-2} \mathrm{di}\end{array}$ & $\begin{array}{l}13.507 \\
2.19^{*} \\
0.242\end{array}$ & $\begin{array}{c}76.2 \\
1.175 \\
1.53\end{array}$ & $\begin{array}{c}12.419 \\
1.316 \\
0.307\end{array}$ & $\begin{array}{c}24.181 \\
1.278 \\
0.59^{*}\end{array}$ & $\begin{array}{c}1.691 \\
0.353 \\
0.043 * *\end{array}$ \\
\hline Pusa Basmati 1 & $\begin{array}{c}\text { Mean } \\
\text { bi } \\
\mathrm{S}^{-2} \mathrm{di}\end{array}$ & $\begin{array}{c}12.159 \\
1.194 \\
1.58^{* *}\end{array}$ & $\begin{array}{c}81.063 \\
1.238 \\
2.508\end{array}$ & $\begin{array}{c}10.219 \\
0.39^{*} \\
0.262\end{array}$ & $\begin{array}{c}25.328 \\
1.188 \\
3.99 * *\end{array}$ & $\begin{array}{c}1.86 \\
1.419 \\
0.039^{*}\end{array}$ \\
\hline
\end{tabular}

Journal of Experimental Biology and Agricultural Sciences http://www.jebas.org 


\begin{tabular}{|c|c|c|c|c|c|c|}
\hline Genotypes & Parameters & Grain Yield & Plant Height & Tiller/ Plant & Panicle Length & Panicle Weight \\
\hline Imp Pusa Bas 1 & $\begin{array}{l}\text { Mean } \\
\text { bi } \\
\mathrm{S}^{-2} \mathrm{di}\end{array}$ & $\begin{array}{c}12.186 \\
1.301 \\
3.13 * *\end{array}$ & $\begin{array}{c}75.002 \\
0.803 \\
15.13 *\end{array}$ & $\begin{array}{c}10.429 \\
0.539 \\
1.97 * *\end{array}$ & $\begin{array}{c}24.982 \\
2.202 \\
3.48 * *\end{array}$ & $\begin{array}{c}1.88 \\
1.42 \\
0.073^{* *}\end{array}$ \\
\hline HKR 98-476 & $\begin{array}{l}\text { Mean } \\
\text { bi } \\
\mathrm{S}^{-2} \mathrm{di}\end{array}$ & $\begin{array}{l}9.842 \\
0.34^{*} \\
0.542\end{array}$ & $\begin{array}{c}104.67 \\
1.45 \\
3.416\end{array}$ & $\begin{array}{c}13.286 \\
1.898 \\
6.23^{* *}\end{array}$ & $\begin{array}{c}24.094 \\
1.385 \\
0.406\end{array}$ & $\begin{array}{c}1.274 \\
0.793 \\
0.042 * *\end{array}$ \\
\hline HKR 3-408 & $\begin{array}{l}\text { Mean } \\
\text { bi } \\
\mathrm{S}^{-2} \mathrm{di}\end{array}$ & $\begin{array}{c}10.636 \\
0.691 \\
2.35^{* *}\end{array}$ & $\begin{array}{c}101.91 \\
0.921 \\
35.77 * *\end{array}$ & $\begin{array}{c}11.743 \\
0.393 \\
2.96^{* *}\end{array}$ & $\begin{array}{c}24.295 \\
0.462 \\
0.75 *\end{array}$ & $\begin{array}{c}1.39 \\
0.13^{*} \\
0.013\end{array}$ \\
\hline HKR 06-434 & $\begin{array}{c}\text { Mean } \\
\text { bi } \\
\mathrm{S}^{-2} \mathrm{di}\end{array}$ & $\begin{array}{c}12.136 \\
1.649 \\
5.27 * *\end{array}$ & $\begin{array}{c}106.071 \\
1.124 \\
15.62 * *\end{array}$ & $\begin{array}{c}10.933 \\
0.587 \\
2.10^{* *}\end{array}$ & $\begin{array}{c}23.714 \\
0.742 \\
0.266\end{array}$ & $\begin{array}{l}1.508 \\
1.342 \\
0.008\end{array}$ \\
\hline HKR 06-443 & $\begin{array}{c}\text { Mean } \\
\text { bi } \\
\mathrm{S}^{-2} \mathrm{di}\end{array}$ & $\begin{array}{c}11.24 \\
0.666 \\
3.92 * *\end{array}$ & $\begin{array}{c}91.748 \\
0.92 \\
22.32 * *\end{array}$ & $\begin{array}{c}12.576 \\
1.309 \\
0.239\end{array}$ & $\begin{array}{c}24.473 \\
0.91 \\
0.025\end{array}$ & $\begin{array}{l}1.362 \\
0.679 \\
0.001\end{array}$ \\
\hline HKR 06-487 & $\begin{array}{c}\text { Mean } \\
\text { bi } \\
\mathrm{S}^{-2} \mathrm{di}\end{array}$ & $\begin{array}{c}11.31 \\
0.851 \\
3.74 * *\end{array}$ & $\begin{array}{c}108.87 \\
0.777 \\
15.33 *\end{array}$ & $\begin{array}{c}10.686 \\
1.28 \\
8.22^{* *}\end{array}$ & $\begin{array}{c}24.783 \\
0.423 \\
4.60 * *\end{array}$ & $\begin{array}{c}1.562 \\
0.551 \\
0.19 * *\end{array}$ \\
\hline HKR 08-417 & $\begin{array}{c}\text { Mean } \\
\text { bi } \\
\mathrm{S}^{-2} \mathrm{di}\end{array}$ & $\begin{array}{c}14.888 \\
1.161 \\
18.60^{* *}\end{array}$ & $\begin{array}{c}91.381 \\
0.874 \\
11.45 *\end{array}$ & $\begin{array}{c}12.629 \\
0.976 \\
2.47 * *\end{array}$ & $\begin{array}{c}24.386 \\
1.097 \\
0.63 *\end{array}$ & $\begin{array}{c}1.825 \\
1.09 \\
0.03^{*}\end{array}$ \\
\hline HKR 08-425 & $\begin{array}{c}\text { Mean } \\
\text { bi } \\
\mathrm{S}^{-2} \mathrm{di}\end{array}$ & $\begin{array}{c}14.249 \\
1.50 * \\
2.57 *\end{array}$ & $\begin{array}{c}89.886 \\
1.205 \\
24.81 * *\end{array}$ & $\begin{array}{c}12.79 \\
0.89 \\
0.842\end{array}$ & $\begin{array}{c}23.87 \\
0.277 \\
2.58 * *\end{array}$ & $\begin{array}{l}1.558 \\
0.986 \\
0.019\end{array}$ \\
\hline Haryana Mahek-1 & $\begin{array}{c}\text { Mean } \\
\text { bi } \\
\mathrm{S}^{-2} \mathrm{di}\end{array}$ & $\begin{array}{c}10.596 \\
0.69 \\
1.57 *\end{array}$ & $\begin{array}{c}96.501 \\
1.039 \\
56.90^{* *}\end{array}$ & $\begin{array}{c}10.314 \\
0.51 \\
7.65 * *\end{array}$ & $\begin{array}{c}24.348 \\
1.004 \\
6.07 * *\end{array}$ & $\begin{array}{c}1.844 \\
0.941 \\
0.033^{*}\end{array}$ \\
\hline Haryana Basmati-1 & $\begin{array}{c}\text { Mean } \\
\text { bi } \\
\mathrm{S}^{-2} \mathrm{di}\end{array}$ & $\begin{array}{c}12.139 \\
1.259 \\
3.36^{* *}\end{array}$ & $\begin{array}{c}95.314 \\
1.084 \\
70.17^{* *}\end{array}$ & $\begin{array}{c}12.029 \\
1.151 \\
4.47^{*} *\end{array}$ & $\begin{array}{c}25.877 \\
1.74^{*} \\
0.183\end{array}$ & $\begin{array}{c}1.925 \\
1.67 * \\
0.041 * *\end{array}$ \\
\hline Travari Basmati & $\begin{array}{l}\text { Mean } \\
\text { bi } \\
\mathrm{S}^{-2} \mathrm{di}\end{array}$ & $\begin{array}{c}12.974 \\
0.917 \\
11.89 * *\end{array}$ & $\begin{array}{c}109.695 \\
1.146 \\
66.11 * *\end{array}$ & $\begin{array}{c}12.452 \\
0.803 \\
0.109\end{array}$ & $\begin{array}{c}24.263 \\
1.344 \\
1.35^{* *}\end{array}$ & $\begin{array}{l}1.508 \\
0.476 \\
0.053\end{array}$ \\
\hline Super Basmati & $\begin{array}{l}\text { Mean } \\
\text { bi } \\
\mathrm{S}^{-2} \mathrm{di}\end{array}$ & $\begin{array}{c}11.883 \\
0.846 \\
7.04 * *\end{array}$ & $\begin{array}{c}98.748 \\
1.053 \\
10.96 *\end{array}$ & $\begin{array}{l}12.01 \\
0.796 \\
1.02 *\end{array}$ & $\begin{array}{c}24.508 \\
0.502 \\
2.72 * *\end{array}$ & $\begin{array}{l}1.511 \\
1.195 \\
0.118\end{array}$ \\
\hline CSR-30 & $\begin{array}{l}\text { Mean } \\
\text { bi } \\
\mathrm{S}^{-2} \mathrm{di}\end{array}$ & $\begin{array}{c}8.740 \\
1.096 \\
7.76^{* *}\end{array}$ & $\begin{array}{c}97.952 \\
1.064 \\
69.22 * *\end{array}$ & $\begin{array}{c}12.257 \\
0.649 \\
1.55^{*}\end{array}$ & $\begin{array}{c}22.658 \\
0.4 \\
3.85^{* * *}\end{array}$ & $\begin{array}{l}1.008 \\
0.74 * \\
0.013\end{array}$ \\
\hline BASMATI-370 & $\begin{array}{l}\text { Mean } \\
\text { bi } \\
\mathrm{S}^{-2} \mathrm{di}\end{array}$ & $\begin{array}{c}12.101 \\
0.44^{*} \\
2.95^{* *}\end{array}$ & $\begin{array}{c}116.067 \\
1.242 \\
174.32^{* *}\end{array}$ & $\begin{array}{c}10.552 \\
0.998 \\
1.85 * *\end{array}$ & $\begin{array}{c}25.29 \\
1.209 \\
6.28 * *\end{array}$ & $\begin{array}{l}1.734 \\
1.001 \\
0.003\end{array}$ \\
\hline PUSA RH-10 & $\begin{array}{c}\text { Mean } \\
\text { bi } \\
\mathrm{S}^{-2} \mathrm{di}\end{array}$ & $\begin{array}{c}15.250 \\
0.405 \\
29.65 * *\end{array}$ & $\begin{array}{c}86.005 \\
0.872 \\
38.75^{* *} *\end{array}$ & $\begin{array}{c}12.1 \\
1.157 \\
2.41 * *\end{array}$ & $\begin{array}{c}24.926 \\
0.499 \\
0.294\end{array}$ & $\begin{array}{c}2.234 \\
0.28 * * \\
0.004\end{array}$ \\
\hline
\end{tabular}

$*$, ** Significant at $5 \%$ and $1 \%$ respectively; bi: Regression coefficient; $\mathrm{S}^{-2} \mathrm{di} .:$ Deviation from regression 


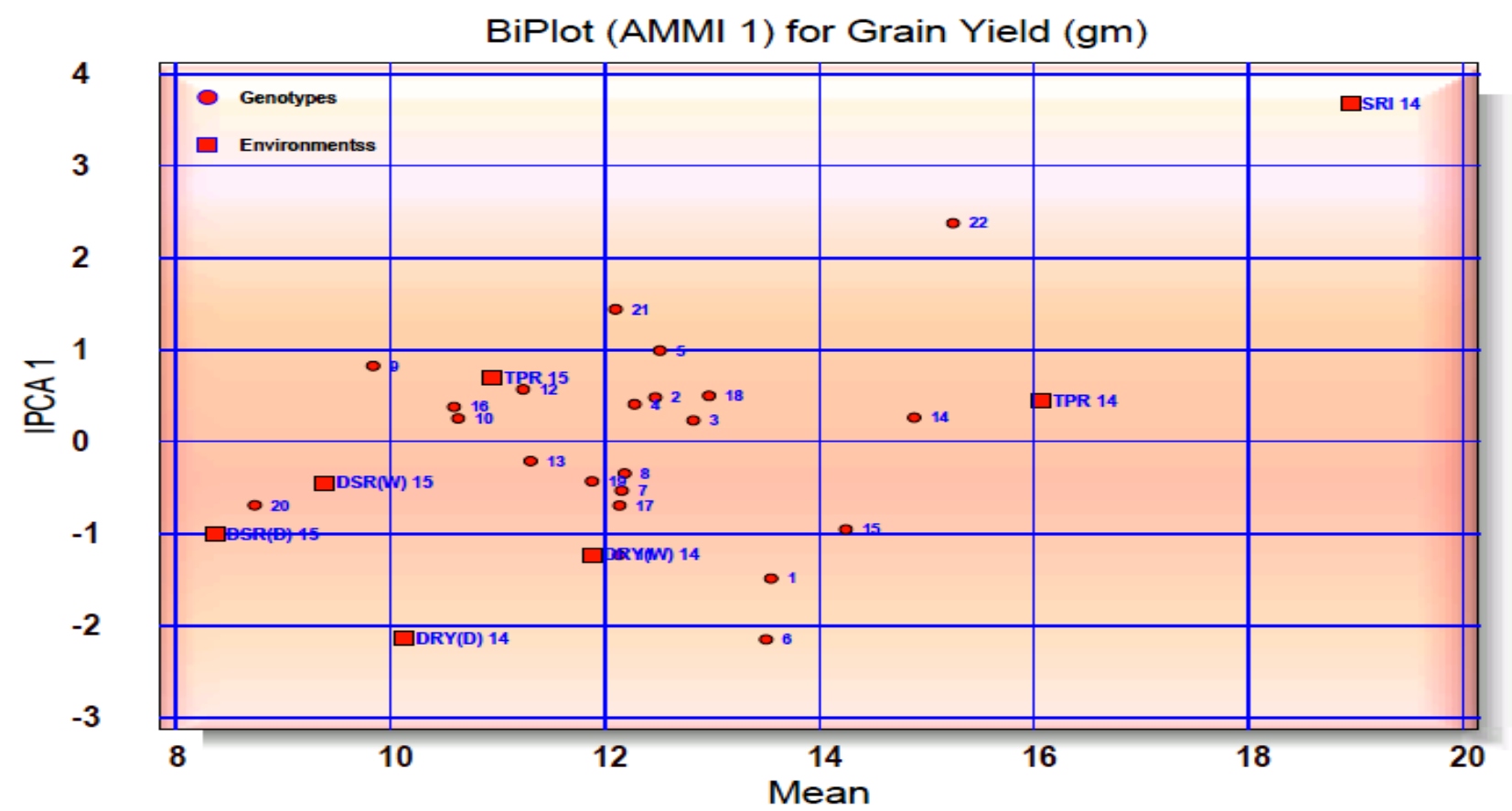

Figure 1 AMMI biplot of grain yield per plant main effects and GxE interaction of 22 rice genotypes under seven environments for grain yield per plant.

that the interaction of the twenty-two rice genotypes with seven environments was clearly predicted by the first two components of PCAI and PCAII. Genotypes HKR 3-408, Haryana Mehak-1, Pusa Sugandh-2, HKR 08-417 differed only in main (additive) effect. Conversely, many the genotypes differed only in interaction effects. While the genotype CSR-30 and Pusa RH-10 differed both in main effect and interaction effect. The interactions of environments were highly varied, while the normal TPR (E1) and TPR (E5) production system had low interaction, SRI (E2) high positive, DSR (wet) (E3), DSR (dry) (E4), DSR (wet) (E6), DSR (dry) (E7) had negative interaction. Production system (TPR and SRI) i.e. environments SRI, TPR had been always on the right-hand side of the midpoint of the main effect axis, seemed to be favorable environments, while DSR (wet) and DSR (dry) were generally less favorable environments. The genotypes having high interaction with environments were adapted to specific environments. Genotypes Pusa RH-10, Travori Basmati, HKR 08-417 with high mean and positive interaction was adapted to the favourable environment. Conversely, the genotype CSR-30 with low mean and negative interaction adapted to unfavourable environments. Pusa RH-10 had a higher mean grain yield and large IPCA1 scores indicated that they were unstable genotypes. Genotypes HKR 06-487, HKR 3-408, Haryana Mehak-1, Pusa Sugandh-2, HKR 08-417 had an IPAC1 score near zero. Hence, had small interaction effects indicating that these varieties were less influenced by the environment thus, stable. HKR 08-417 was found to be well acclimatized to the environments studied in this study. Using Figure the genotype and environment combination can be predicated as defined by Zobel et al. (1988).

The environments of the normal production system (E1) and DSR (Wet) (E7) had short spokes and they did not exert strong interactive force while environment SRI (E2), DSR (W) (E3), DSR (D) (E4), DSR (D) (E6), TPR (E5) having long spoke exert strong interaction. AMMI biplot for grain yield per plant of 22 rice genotypes studied under seven environments is shown in the Figure 1. Genotypes Pusa RH-10, Imp Pusa Basmati 1, HKR 98476 were away from thus, most responsive. In this case, the bestadapted genotypes with respect to sites SRI were Pusa RH-10 but unsuitable for DSR (dry), DSR (wet). Genotype Imp Pusa Basmati1, Haryana Mehak-1 were adapted to DSR (wet) (E3) and CSR-30, Haryana Basmati-1 to DSR (wet) (E7) while Super Basmati, HKR 06-487 to DSR (dry) (E6). Genotypes HKR 98476, Haryana Mehak-1, HKR 06--443, HKR 3-408 adapted to TPR(E5) and Pusa Sugandh 3 to TPR (E1). In Figure 2 the environment effects are joined to the origin, the sites with strong interaction are those with long spoke while week interactive force were present in the sites with short spokes. For effective breeding and adaption in different environmental conditions required stable 


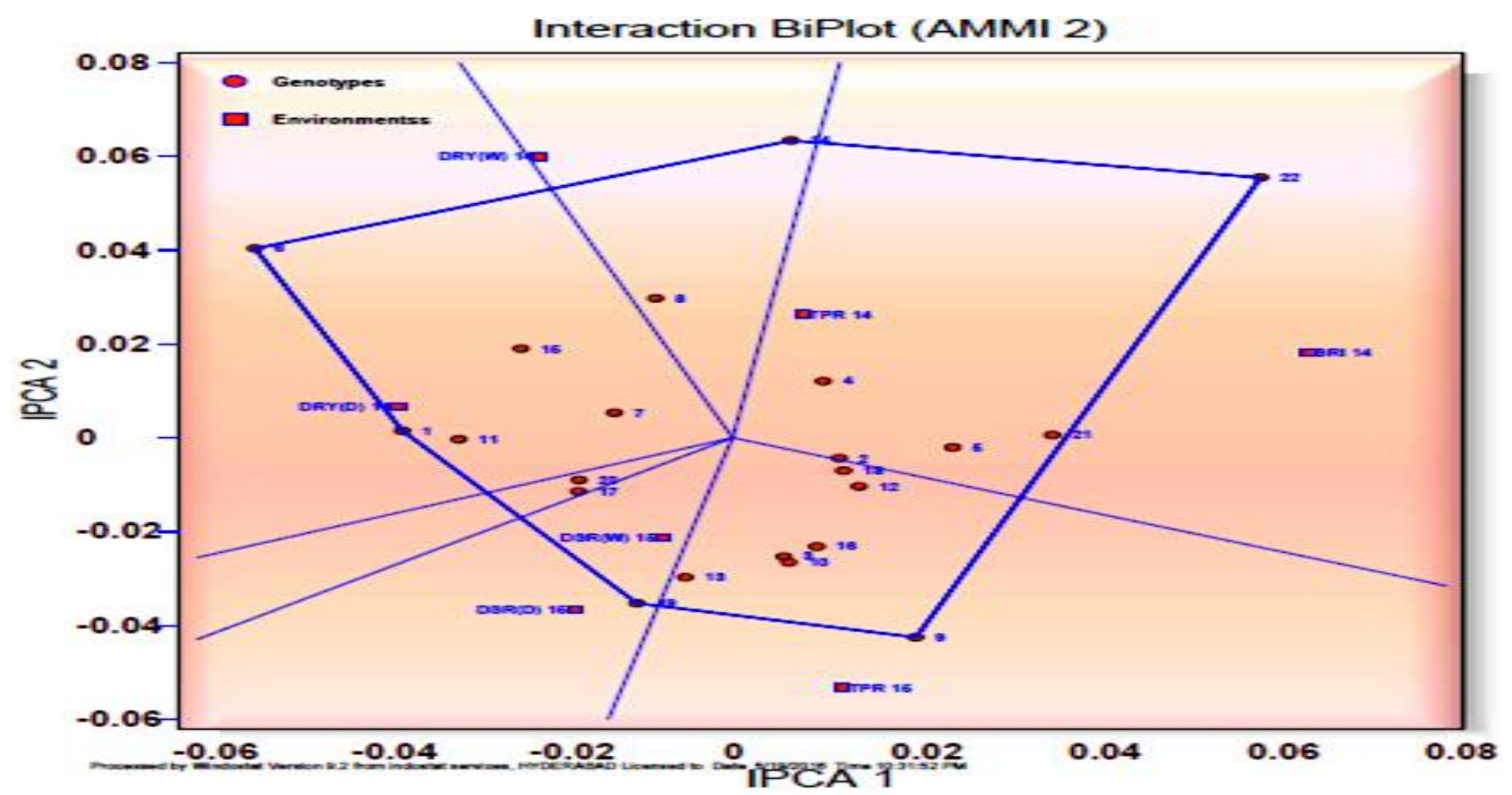

Figure 2 AMMI-2 model for grain yield per plant showing IPCA scores of rice genotype planted across environment (E).

Legend : 1: Pusa Basmati 1121, 2: Pusa Basmati 1509, 3: Pusa Sugandh 2, 4: Pusa Sugandh3, 5: Pusa Sugandh 5, 6: Pusa Basmati 6, 7: Pusa Basmati 1, 8: Imp Pusa Basmati 1, 9: HKR 98-476, 10: HKR 3-408, 11: HKR06-434, 12: HKR 06-443, 13: HKR 06-487, 14: HKR 08417,15:HKR 08-425, 16: Haryana mehak-1, 17: Haryana Basmati-1, 18: Traori Basmati, 19: Super Basmati, 20: CSR-30, 21: Basmati 370, 22: Pusa RH 10, E1: TPR 14,E2: SRI14, E3: DSR(wet)14, E4: DSR (dry)14, E5:TPR15, E6 : DSR(D)15, E7: DSR(W)15.

and GEI study (Liang et al., 2015). GEI is analyzed by regression and multivariate methods. The benefits of methods based on multivariate analysis are naturally more accurate concept of GEI, the simplicity of interpretation of results delivered using Biplots charts (Carvalh et al. 2015). AMMI1 and AMMI2 biplot in mutienvironment analysis and genotype evaluation because it clarifies more G+GE (Agyeman et al., 2015).

\section{Acknowledgements}

The author is thankful to the Regional Director RRS, Kaul, CCSHAU, Haryana for providing land, inputs and other facilities required for conducting the above experiment.

\section{Disclosure statement}

Authors declare that no conflict of interest could arise.

\section{Conflicts of interest}

All authors declare that there exist no commercial or financial relationships that could in any way lead to a potential conflict of interest.

\section{References}

Agyeman A, Parkes E, Peprah BB (2015) Ammi and GGE biplot analysis of root yield performance of cassava genotypes in the forest and coastal ecologies. International Journal of Agricultural Policy and Research 3: 222-232.

Amiri R, Bahraminejad S, Sasani S, Jalali-Honarmand S, Fakhri R (2015) Bread wheat genetic variation for grain's protein, iron and zinc concentrations as uptake by their genetic ability. European Journal of Agronomy 67: 20-26.

Carvalh LCB, Damascenosilva KJ, Rocha MDM, Oliveira GCX (2015) Evolution of Methodology for the Study of Adaptability and Stability in Cultivated Species. African Journal of Agricultural Research 11: 990-1000.

Dawe D (2010) The Rice Crises. Markets, Policy and Food Security. 1st Edition. Published by The Food Agricultural Organization and Earthscan, London, Washington, DC. Pp.368.

Eberhart SA, Russel WA (1966) Stability parameters for comparing varieties. Crop Sciences 6: 36-40. 
Finlay KW, Wilkinson GN (1963) The analysis of adaptation in a plant breeding programmed. Australian Journal Agriculture Research 14: 42-754.

Fuller D, Weisskopf A, Castillo C (2016) Pathways of Rice Diversification across Asia. Archaeology International 19: 84-96. DOI:https://doi.org/10.5334/ai.1915.

Gauch Jr HG, Zobel RW (1989) Accuracy and selection success in yield trials analysis. Theoretical and Applied Genetics 77: 443481 .

Ghritlahre SK, Sarial AK, Ram MR, Aneja DR, Singh R (2012) Genetic yield potential of rice (Oryza sativa L.)through water saving and high yielding SRI technology. Indian Journal of Agriculture Science 82: 260-263

Jain BT, Sarial AK, Hari Kesh (2018) Comparison of Yield Potential of Basmati Rice (Oryza sativa) through Direct Seeded Rice, SRI and Conventional Technology Research. Journal of Agricultural Sciences 9: 300-306

Liang S, Ren G, Liu J, Zhao X, Zhou M, McNeil D, Ye G (2015) Genotype by environment interaction is important for grain yield in irrigated lowland rice. Field Crops Research 180: 90-99.

Miro B, Ismail AM (2013) Tolerance of anaerobic conditions caused by flooding during germination and early growth in rice (Oryza sativa L.). Frontiers in Plant Science 4:269. doi: 10.3389/fpls.2013.00269

Mohammadi M, Karimizadeh R, Hosseinpour T, Falahi HA, Khanzadeh H, SabaghniaN (2012) Genotype $\times$ Environment interaction and stability analysis of seed yield of durum wheat genotypes in dryland conditions. Notulae Scientia Biologicae 4: 57-64. DOI: 10.15835/nsb.4.3.7807.

Nguyen N, Ferrero A (2006) Meeting the challenges of global rice production. Paddy and Water Environment 4: 1-9. DOI: https://doi.org/10.1007/s10333-005-0031-5.

Pandey S, Velasco L (2002) Economics of direct seeding in Asia: patterns of adoption andresearch priorities in Direct seeding: Researchstrategies and opportunities. IRRI, Philippines 3-14.

Panse VG, Sukhatme PV (1985) Statistical Methods for Agricultural Workers, 2nd edition, Indian Council of Agricultural Research Publication, New Delhi.

Prasetyo D, Anindita AR (2016) Import Demand Function Of Rice InIndonesia. Journal of Social Economic. 27: 1-6.

Rakshit S, Ganapathy KN, Gomashe SS, Rathore A, Ghorade RB,
Nagesh Kumar MV, Ganesmurthy K, Jain SK, Kamtar MY, Sachan JS, Ambekar SS, Ranwa BR, Kanawade DG, Balusamy M, Kadam D, Sarkar A, Tonapi,VA, Patil JV (2012) GGE biplot analysis to evaluate genotype, environment and their interactions in sorghum multi-locationdata. Euphytica 185:465-479. doi:10.1007/s10681-012-0648-6

Raman A, Ladha JK, Kumar V, Sharma S, Piepho HP (2011) Stability analysis of farmer participatory trials for conservationagriculture using mixed models. Field Crops Research 121: 450-459.

Rao AN, Wani SP, Ramesha MS, Ladha JK (2017) Rice production systems in Rice production worldwide. Springer International Publishing, ISBN 978-3-319-47516-5 .

Satyanarayana A, Thiyagarajan TM, Uphoff N (2007)Opportunities for water saving with higher yield from the system of rice intensification. Irrigation Science 25: 99-115.

Sinha SK, Biswas S (1987) Adaptability of rice varieties during boro season of west Bengal. Indian Agriculture 31: 15-18.

Surendra Kumar (2010) Studies on genotypic performance and genotype $\mathrm{x}$ environment interaction in rice in different production systems, M. Sc. Thesis submitted to the CCS HAU, Hisar.

Takashima S, Oyoshi K, Fukuda T, Okumura T, Tomiyama N, Nagano T (2013) Asia Rice Crop Monitoring in GEO GLAM. 2013 Second International Conference on Agro-Geoinformatics (Agro-Geoinformatics) Pp. 398-401, https://doi.org/10.1109/Argo-Geoinformatics.2013.6621951.

Uphoff N (2003) SRI gives higher yields with fewer external input? the system of rice intensification and potential contributions to agricultural sustainability. Indian Journal of Agricultural Sustainability 1:38-50.

Vaughan D, Lu BR, Tomooka N (2008) The evolving story of rice evolution. Plant Science 174: 394-408, https://doi.org/10.1016/j.plantsci.2008.01.016.

Yates F, Cochran WG (1938) The analysis of groups of experiments. Journal of Agricultural Science28: 556-580.

Yoshida S, Forno DA, Cock JH, Gomez KA (1976) Laboratory manual for physiological studies of rice. $\left(3^{\text {rd }} \mathrm{Ed}\right.$.), International Rice Research Institute, Los Banos, Philippines.

Zobel RW, Wright MJ, Gauch HG (1988) Statistical analysis of a yield trial. Agronomy Journal 80: 388-393. 